Bull. Korean Math. Soc. 50 (2013), No. 1, pp. 11-24

http://dx.doi.org/10.4134/BKMS.2013.50.1.011

\title{
RESOLUTIONS AND DIMENSIONS OF RELATIVE INJECTIVE MODULES AND RELATIVE FLAT MODULES
}

\author{
Yuedi Zeng And JiAnlong Chen
}

\begin{abstract}
Let $m$ and $n$ be fixed positive integers and $M$ a right $R$ module. Recall that $M$ is said to be $(m, n)$-injective if $\operatorname{Ext}^{1}(P, M)=0$ for any $(m, n)$-presented right $R$-module $P ; M$ is said to be $(m, n)$-flat if $\operatorname{Tor}_{1}(N, P)=0$ for any $(m, n)$-presented left $R$-module $P$. In terms of some derived functors, relative injective or relative flat resolutions and dimensions are investigated. As applications, some new characterizations of von Neumann regular rings and p.p. rings are given.
\end{abstract}

\section{Introduction}

Let $\mathscr{C}$ be a class of left $R$-modules and $M$ a left $R$-module. Following ([7]), we say that a homomorphism $\varphi: M \rightarrow C$ is a $\mathscr{C}$-preenvelope of $M$ if $C \in \mathscr{C}$ and the abelian group homomorphism $\operatorname{Hom}\left(\varphi, C^{\prime}\right): \operatorname{Hom}\left(C, C^{\prime}\right) \rightarrow \operatorname{Hom}\left(M, C^{\prime}\right)$ is surjective for each $C^{\prime} \in \mathscr{C}$. A $\mathscr{C}$-preenvelope $\varphi: M \rightarrow C$ is called a $\mathscr{C}$-envelope if every endomorphism $f: C \rightarrow C$ such that $f \varphi=\varphi$ is an isomorphism. A $\mathscr{C}$-envelope $\varphi: M \rightarrow C$ is said to have the unique mapping property (see [6]) if for any homomorphism $f: M \rightarrow C^{\prime}$ with $C^{\prime} \in \mathscr{C}$, there is a unique homomorphism $g: C \rightarrow C^{\prime}$ such that $g \varphi=f$. Dually, we have the definitions of $\mathscr{C}$-precovers and $\mathscr{C}$-covers. $\mathscr{C}$-envelopes $(\mathscr{C}$-covers) may not exist in general, but if they exist, they are unique up to isomorphisms.

Let $m$ and $n$ be fixed positive integers.

A right $R$-module $P$ is said to be $(m, n)$-presented $([17])$ if there exists a right $R$-module exact sequence $0 \rightarrow K \rightarrow R^{m} \rightarrow P \rightarrow 0$, where $K$ is $n$-generated.

A right $R$-module $M$ is said to be $(m, n)$-injective ([4]) if $\operatorname{Ext}^{1}(P, M)=0$ for any $(m, n)$-presented right $R$-module $P$.

A left $R$-module $N$ is said to be $(m, n)$-flat $([17])$ if $\operatorname{Tor}_{1}(P, N)=0$ for any $(m, n)$-presented right $R$-module $P$.

Received August 1, 2010

2010 Mathematics Subject Classification. 16D50, 16E50, $16 \mathrm{E} 10$.

Key words and phrases. $(m, n)$-coherent ring, $(m, n)$-injective module, $(m, n)$-flat module, (pre)cover, (pre)envelope.

The work was supported by the National Natural Science Foundation of China (10971024), the Specialized Research Fund for the Doctoral Program of Higher Education (200802860024) and the Foundation of the Fujian education (JA11209). 
A ring $R$ is called right $(m, n)$-coherent ([17]) in case each $n$-generated submodule of the right $R$-module $R^{m}$ is finitely presented.

Throughout this paper, $R$ is an associative ring with identity and all modules are unitary. We write ${ }_{R} \mathcal{M}\left(\mathcal{M}_{R}\right)$ to indicate the class of all left (right) $R$ modules. For an $R$-module $M, M^{+}=\operatorname{Hom}_{Z}(M, Q / Z)$ denotes the character module of $M$ and $E(M)$ is the injective envelope of $M$. As usual, we denote by id $M, \operatorname{pd} M$ and $\mathrm{fd} M$ the injective dimension, the projective dimension and the flat dimension of $M$, respectively. $\mathscr{A}_{(m, n)}(\mathscr{A})$ and $\mathscr{F}_{(m, n)}(\mathscr{F})$ stand for $(m, n)$-injective (P-injective, i.e., $(1,1)$-injective) right $R$-modules and $(m, n)$ flat (P-flat, i.e., (1,1)-flat) left $R$-modules, respectively.

Recently, $(m, n)$-injective modules and $(m, n)$-flat modules were introduced and studied by many authors (see, for example, $[4,15,17,12,13]$ etc.). Note that every left $R$-module over a right $(m, n)$-coherent $\operatorname{ring} R$ admits an $(m, n)$ flat cover and an $(m, n)$-flat preenvelope (see [12]). In Section 2, it is shown that every right $R$-module over a right $(m, n)$-coherent ring $R$ admits an $(m, n)$ injective cover and an $(m, n)$-injective preenvelope. It is proved that: for a right $(m, n)$-coherent $\operatorname{ring} R$, left $\mathscr{F}_{(m, n)}$ - $\operatorname{dim} N \leq k$ if and only if for every right $\mathscr{F}_{(m, n)}$-resolution $0 \rightarrow M \rightarrow F^{0} \rightarrow F^{1} \rightarrow \cdots$ of any $(n, m)$-presented left $R$-module $M, \operatorname{Hom}\left(F^{k}, N\right) \rightarrow \operatorname{Hom}\left(L^{k}, N\right) \rightarrow 0$ is exact, where $L^{k}=$ $\operatorname{coker}\left(F^{k-2} \rightarrow F^{k-1}\right)$. Then we get [13, Theorem 4.7 and Corollary 4.8], and we only inspect cyclical left $R$-modules in this theorem.

If $R$ is right $(m, n)$-coherent, then $-\otimes-$ on $\mathcal{M}_{R} \times{ }_{R} \mathcal{M}$ is right balanced by $\mathscr{A}_{(m, n)} \times \mathscr{F}_{(m, n)}$ with right derived functors $\mathrm{T}^{t}(-,-) ; \operatorname{Hom}(-,-)$ is left balanced on ${ }_{R} \mathcal{M} \times{ }_{R} \mathcal{M}$ by $\mathscr{F}_{(m, n)} \times \mathscr{F}_{(m, n)}$ with left derived functors $\operatorname{Ex}_{t}(-,-)$; $\operatorname{Hom}(-,-)$ is left balanced on $\mathcal{M}_{R} \times \mathcal{M}_{R}$ by $\mathscr{A}_{(m, n)} \times \mathscr{A}_{(m, n)}$ with left derived functors $\mathrm{E}_{t}(-,-)$. Note that over right generalized morphic rings $R$, each $R / R a$ have a right $\mathbb{R}$-resolution (see Lemma 3.2). Hence, in Section 3, over right generalized morphic rings, the $\mathrm{P}$-injective and $\mathrm{P}$-flat dimensions can be characterized by these derived functors (see Theorem 3.14).

Theorem. Let $R$ be a right generalized morphic ring and $t \geq 2$. Then the following are equivalent.

(1) gl right $\mathscr{A}-\operatorname{dim} \mathcal{M}_{R} \leq t$.

(2) gl left $\mathscr{F}-\operatorname{dim}_{R} \mathcal{M} \leq t$.

(3) gl left $\mathscr{A}-\operatorname{dim} \mathcal{M}_{R} \leq t-2$.

(4) right $\mathscr{F}-\operatorname{dim} R / R a \leq t-2$ for all $a \in R$.

(5) right $\mathscr{A}-\operatorname{dim} R / a R \leq t$ for all $a \in R$.

(6) right $\mathscr{A}-\operatorname{dim} M \leq t$ for all reduced $D$-injective right $R$-module $M$.

(7) $\operatorname{Ex}_{t-1}(M, N)=0$ for all $M, N \in_{R} \mathcal{M}$ and all $k \geq-1$.

(8) $\operatorname{Ex}_{t-1}(M, N)=0$ for all $M, N \in_{R} \mathcal{M}$.

(9) $\operatorname{Ex}_{t-1}(M, N)=0$ for all $N \in_{R} \mathcal{M}$ and all Warfield cotorsion left $R$ module $M$.

(10) $\operatorname{Ex}_{t-1}(R / R a, N)=0$ for all $N \in_{R} \mathcal{M}$ and all $a \in R$.

(11) $\operatorname{Ex}_{t-1}(R / R a, R / R b)=0$ for all $a, b \in R$. 
(12) $\mathrm{T}^{t+k}(M, N)=0$ for all $M \in \mathcal{M}_{R}, N \in_{R} \mathcal{M}$ and all $k \geq-1$.

(13) $\mathrm{T}^{t}(M, N)=\mathrm{T}^{t-1}(M, N)=0$ for all $M \in \mathcal{M}_{R}, N \in \in_{R} \mathcal{M}$.

(14) $\mathrm{T}^{t}(R / a R, N)=\mathrm{T}^{t-1}(R / a R, N)=0$ for all $N \in_{R} \mathcal{M}$ and all $a \in R$.

(15) $\mathrm{T}^{t}(M, R / R b)=\mathrm{T}^{t-1}(M, R / R b)=0$ for all $M \in \mathcal{M}_{R}$ and all $b \in R$.

(16) $\mathrm{T}^{t}(R / a R, R / R b)=\mathrm{T}^{t-1}(R / a R, R / R b)=0$ for all $a, b \in R$.

(17) $\mathrm{E}_{t+k}(M, N)=0$ for all $M, N \in \mathcal{M}_{R}$ and all $k \geq-1$.

(18) $\mathrm{E}_{t-1}(M, N)=0$ for all reduced $D$-injective right $R$-module $M$ and all $N \in \mathcal{M}_{R}$.

(19) $\mathrm{E}_{t-1}(R / a R, M)=0$ for all reduced $D$-injective right $R$-module $M$ and all $a \in R$

(20) right $\operatorname{Proj}-\operatorname{dim} R / a R \leq t-2$ for all $a \in R$.

(21) $H$ is a direct summand of $R_{t-2}$ for any right $\mathbb{R}$-resolution of $R / R a$ and any $a \in R$, where $H=\operatorname{ker}\left(R_{t-2} \rightarrow R_{t-1}\right)$.

(22) $\operatorname{Ext}^{t+1}(R / a R, M)=0$ for all $M \in \mathcal{M}_{R}$ and all $a \in R$.

(23) $\operatorname{Tor}_{t+1}(R / a R, N)=0$ for all $N \in_{R} \mathcal{M}$ and all $a \in R$.

(24) $\mathrm{fd}(R / a R) \leq t$.

(25) $\operatorname{pd}(R / a R) \leq t$.

Hence we show that (1) and (2) in [13, Theorem 4.12] are equivalent. For $t=0, R$ is a von Neumann regular ring; for $t=1, R$ is a right p.p. ring; for $t=2, r(a)$ is cyclical generated and projective for any $a \in R$. Some new descriptions of these rings are given in this section.

\section{Resolutions and dimensions}

Following [8, Proposition 8.4.1], the left $\mathscr{C}$-dimension of a left $R$-module $M$, denoted by left $\mathscr{C}-\operatorname{dim} M$, is defined as $\inf \{m:$ there is a left $\mathscr{C}$-resolution of the form $0 \rightarrow F_{m} \rightarrow \cdots \rightarrow F_{0} \rightarrow M \rightarrow 0$ of $\left.M\right\}$; the right $\mathscr{C}$-dimension of a left $R$-module $M$, denoted by right $\mathscr{C}-\operatorname{dim} M$, is defined as $\inf \{m$ : there is a right $\mathscr{C}$-resolution of the form $0 \rightarrow M \rightarrow F^{0} \rightarrow \cdots \rightarrow F^{m} \rightarrow 0$ of $\left.M\right\}$. If there is no such $m$, set left (right) $\mathscr{C}$ - $\operatorname{dim} M=\infty$. The global left (right) $\mathscr{C}$-dimension of ${ }_{R} \mathcal{M}$, denoted by $g l$ left (right) $\mathscr{C}-\operatorname{dim}_{R} \mathcal{M}$, is defined to be $\sup \left\{\right.$ left (right) $\left.\mathscr{C}-\operatorname{dim} M: M \in_{R} \mathcal{M}\right\}$. Similarly, we have these definitions for right $R$-modules. In this section, we consider $(m, n)$-injective and $(m, n)$-flat resolutions and dimensions.

Recall that, given a left $R$-module $U$ with submodule $U_{0}, U_{0}$ is called $(m, n)$ pure in $U$ if the canonical map $P \otimes U_{0} \rightarrow P \otimes U$ for any $(m, n)$-presented right $R$-module, or equivalently, for every $(n, m)$-presented left $R$-module $V$, the canonical map $\operatorname{Hom}(V, U) \rightarrow \operatorname{Hom}\left(V, V / U_{0}\right) \rightarrow 0$ is exact (see [19, Definition 1.3 and Theorem 1.5]). Similarly, we have the definition of right $R$-modules.

In view of [12, Theorem 2.3], if $R$ is a right $(m, n)$-coherent ring, then every left $R$-module has an $(m, n)$-flat preenvelope and an $(m, n)$-flat cover.

Theorem 2.1. Let $R$ be a right $(m, n)$-coherent ring, $k$ a nonnegative integer and $N$ a left $R$-module. Then the following are equivalent.

(1) left $\mathscr{F}_{(m, n)}-\operatorname{dim} N \leq k$. 
(2) For every right $\mathscr{F}_{(m, n)}$-resolution $0 \rightarrow M \rightarrow F^{0} \rightarrow F^{1} \rightarrow \cdots$ of any left $R$-module $M, \operatorname{Hom}\left(F^{k}, N\right) \rightarrow \operatorname{Hom}\left(L^{k}, N\right) \rightarrow 0$ is exact, where $L^{k}=$ $\operatorname{coker}\left(F^{k-2} \rightarrow F^{k-1}\right)$.

(3) For every right $\mathscr{F}_{(m, n)}$-resolution $0 \rightarrow M \rightarrow F^{0} \rightarrow F^{1} \rightarrow \cdots$ of any $(n, m)$-presented left $R$-module $M, \operatorname{Hom}\left(F^{k}, N\right) \rightarrow \operatorname{Hom}\left(L^{k}, N\right) \rightarrow 0$ is exact, where $L^{k}=\operatorname{coker}\left(F^{k-2} \rightarrow F^{k-1}\right)$.

Proof. $(2) \Rightarrow(3)$ is trivial.

We proceed by induction. Let $0 \rightarrow K \rightarrow F \rightarrow N \rightarrow 0$ be an exact sequence where $F \rightarrow N$ is an $\mathscr{F}_{(m, n)}$-cover and $\operatorname{Ext}^{1}(G, K)=0$ for all left $R$-module $G \in \mathscr{F}_{(m, n)}$ by [16, Lemma 2.1.1].

Suppose $k=0$. (1) $\Rightarrow(2)$ is clear.

$(3) \Rightarrow(1)$. Let $M$ be any $(n, m)$-presented left $R$-module. Consider the following commutative diagram:

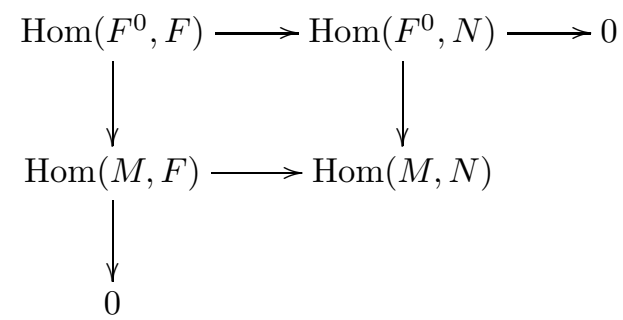

Since $\operatorname{Hom}\left(F^{0}, N\right) \rightarrow \operatorname{Hom}(M, N) \rightarrow 0$ is exact by (3),

$$
\operatorname{Hom}(M, F) \rightarrow \operatorname{Hom}(M, N) \rightarrow 0
$$

is exact. This means that $K$ is $(m, n)$-pure in $F$, that is, $0 \rightarrow H \otimes K \rightarrow H \otimes F$ is exact for any right $R$-module $(m, n)$-presented $H$. Then we have an exact sequence: $\operatorname{Tor}_{1}(H, F) \rightarrow \operatorname{Tor}_{1}(H, N) \rightarrow H \otimes K \rightarrow H \otimes F$. It follows that $\operatorname{Tor}_{1}(H, N)=0$, and hence $N \in \mathscr{F}_{(m, n)}$.

Suppose $k>0$. If left $\mathscr{F}_{(m, n)}-\operatorname{dim} K \leq k-1$, then left $\mathscr{F}_{(m, n)}-\operatorname{dim} N \leq k$. On the other hand, if left $\mathscr{F}_{(m, n)}-\operatorname{dim} N \leq k$, then there is a left $\mathscr{F}_{(m, n)}$-resolution of $M: 0 \rightarrow F_{k} \rightarrow \cdots \rightarrow F_{0} \rightarrow M \rightarrow 0$, where $K_{0}=\operatorname{ker}\left(F_{0} \rightarrow M\right)$. Since $F \rightarrow N$ is an $\mathscr{F}_{(m, n)}$-cover, $F_{0} \cong F \oplus H_{0}$ and $K_{0} \cong K \oplus H_{0}$. This implies that left $\mathscr{F}_{(m, n)}-\operatorname{dim} K \leq k-1$.

Consider the following commutative diagrams:

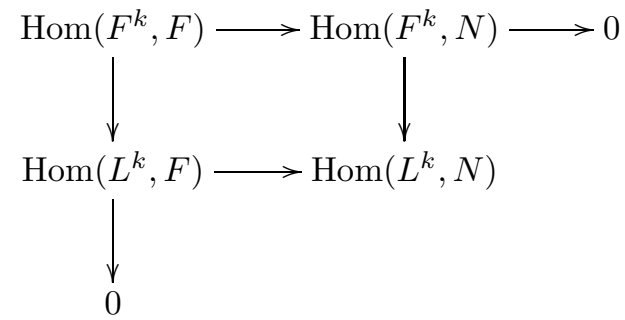


and

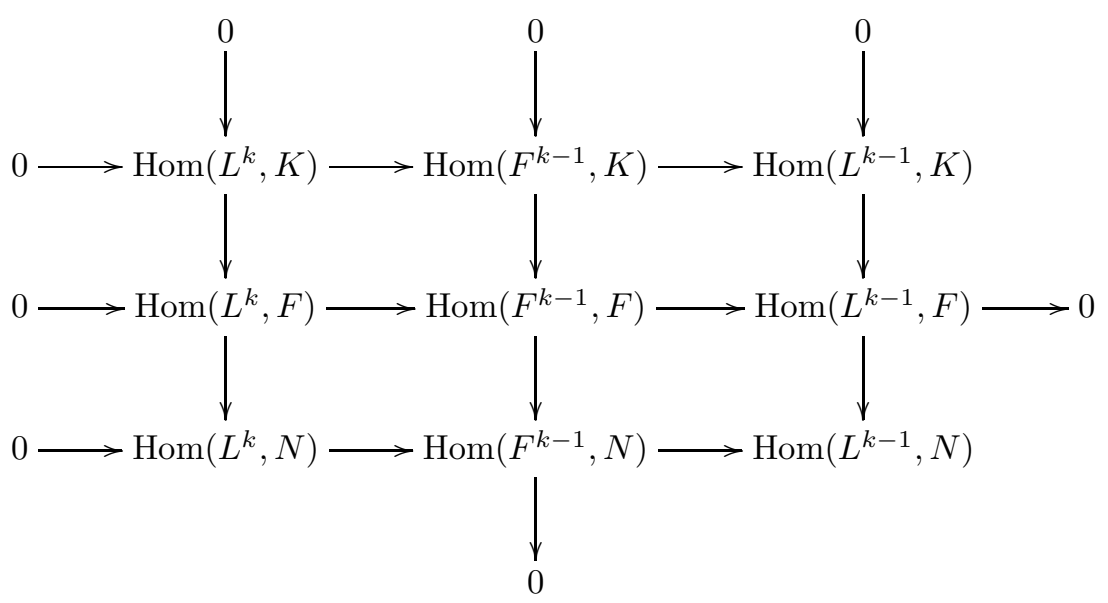

Hence left $\mathscr{F}_{(m, n)}-\operatorname{dim} N \leq k$ if and only if left $\mathscr{F}_{(m, n)}-\operatorname{dim} K \leq k-1$ if and only if $\operatorname{Hom}\left(F^{k-1}, K\right) \rightarrow \operatorname{Hom}\left(L^{k-1}, K\right) \rightarrow 0$ is exact by induction if and only if $\operatorname{Hom}\left(L^{k}, F\right) \rightarrow \operatorname{Hom}\left(L^{k}, N\right) \rightarrow 0$ is exact by the second diagram if and only if $\operatorname{Hom}\left(F^{k}, N\right) \rightarrow \operatorname{Hom}\left(L^{k}, N\right) \rightarrow 0$ is exact by the first diagram.

Corollary 2.2. The following are equivalent for a right $(m, n)$-coherent ring $R$ and an integer $k \neq 0$ :

(1) left $\mathscr{F}_{(m, n)}-\operatorname{dim} R_{R}^{+} \leq k$;

(2) every right $\mathscr{F}_{(m, n)}$-resolution $0 \rightarrow M \rightarrow F^{0} \rightarrow F^{1} \rightarrow \cdots$ of any left $R$-module $M$ is exact at $F^{i}$ for every $i \geq k-1$;

(3) every right $\mathscr{F}_{(m, n)}$-resolution $0 \rightarrow M \rightarrow F^{0} \rightarrow F^{1} \rightarrow \cdots$ of any $(n, m)$ presented left $R$-module $M$ is exact at $F^{i}$ for every $i \geq k-1$;

(4) left $\mathscr{F}_{(m, n)}-\operatorname{dim} N \leq k$, where $N$ is any injective cogenerator in ${ }_{R} \mathcal{M}$.

Proof. $(2) \Rightarrow(3)$ is trivial.

$(1) \Rightarrow(2)$. By Theorem 2.1, $\operatorname{Hom}\left(F^{k}, R^{+}\right) \rightarrow \operatorname{Hom}\left(L^{k}, R^{+}\right)$is an epimorphism. So $L^{k} \rightarrow F^{k}$ is a monomorphism. It means that $F^{k-2} \rightarrow F^{k-1} \rightarrow F^{k}$ is exact. In addition, left $\mathscr{F}_{(m, n)}-\operatorname{dim} R^{+} \leq t$ for any $t \geq k+1$ by (1), and hence (2) holds.

$(3) \Rightarrow(1)$ holds by Theorem 2.1 .

$(4) \Leftrightarrow(3)$ holds by the similar proof of $(1) \Leftrightarrow(3)$.

Remark 2.3. Clearly, we get [13, Theorem 4.7 and Corollary 4.8]. In particular, we only inspect cyclical left $R$-modules in the preceding results.

Proposition 2.4. If $R$ is right $(m, n)$-coherent, then every right $R$-module admits an $(m, n)$-injective cover.

Proof. Let $F$ be an $(m, n)$-injective right $R$-module and $0 \rightarrow L \rightarrow F \rightarrow F / L \rightarrow$ 0 be a pure exact sequence. This induced a split exact sequence $0 \rightarrow(F / L)^{+} \rightarrow$ 
$F^{+} \rightarrow L^{+} \rightarrow 0$. Thus $(F / L)^{+}$is $(m, n)$-flat since $F^{+}$is $(m, n)$-flat by $[17$, Theorem 5.7]. So $F / L$ is $(m, n)$-injective again by [17, Theorem 5.7]. Since $\mathscr{A}_{(m, n)}$ is closed under direct limits for a right $(m, n)$-coherent ring by $[17$, Theorem 5.7], every right $R$-module has an $\mathscr{F}$-cover by [11, Theorem 2.5].

Now we get the following corollary as in [13, Theorem 2.10].

Corollary 2.5. If $R$ is right $(1,1)$-coherent, then every right $R$-module admits a P-injective cover.

Corollary 2.6. The following are equivalent for a right $(m, n)$-coherent ring $R$ :

(1) $R_{R}$ is $(m, n)$-injective;

(2) $R_{R}^{+}$is $(m, n)$-flat;

(3) Every right $R$-module has an epic $\mathscr{A}_{(m, n)}$-cover;

(4) Every left $R$-module has a monic $\mathscr{F}_{(m, n)}$-preenvelope;

(5) Every $(n, m)$-presented left $R$-module has a monic $\mathscr{F}_{(m, n)}$ (projective)preenvelope;

(6) Every $(n, m)$-presented left $R$-module embeds in a free left $R$-module.

Proof. (1) $\Leftrightarrow(2)$ follows from [17, Theorem 5.7]. (2) $\Leftrightarrow(4) \Leftrightarrow$ (5) come from Corollary 2.2. $(5) \Leftrightarrow(6)$ is trivial.

$(1) \Rightarrow(3)$. Let $M$ be a right $R$-module. Then $M$ has an $\mathscr{A}_{(m, n)}$-cover $g$. On the other hand, there is an exact sequence $F \rightarrow M \rightarrow 0$ with $F$ free. Since $F$ is $(m, n)$-injective by $(1), g$ is an epimorphism.

$(3) \Rightarrow(1)$. Let $f: N \rightarrow R$ be an epic $\mathscr{A}_{(m, n)}$-cover of $R$. Then $R_{R}$ is isomorphic to a direct summand of $N$, and so $R_{R}$ is $(m, n)$-injective.

\section{Derived functors}

In ([18]), a ring $R$ is right generalized morphic if, for every $a \in R$, there is $b \in R$ with $r(a) \cong R / b R(r(a)=\{s \in R: a s=0\})$; in ([13]), a ring $R$ is right strongly $\mathrm{P}$-coherent if every principal right ideal of $R$ is cyclically presented. Examples of right generalized morphic rings include not only von Neumann regular rings, p.p. rings and domains, but also right morphic rings (a ring $R$ is called right morphic by [14], if $r(a) \cong R / a R$ for every $a \in R$ ). Clearly, right generalized morphic ring is right strongly $\mathrm{P}$-coherent. It is easy to see that if $R$ is commutative, then generalized morphic rings and strongly $\mathrm{P}$-coherent rings are the same. A right generalized morphic ring may not be left generalized morphic (the example traces back to [18, Example 2.6]. Clearly, any right generalized morphic ring is right $(1,1)$-coherent. But a right $(1,1)$ coherent ring may not be right generalized morphic. Let $R=\mathcal{Z}_{4} C_{2}$ be a group ring, where $C_{2}=\{1, g\}$ is a group. Since $R$ is coherent, $R$ is right generalized morphic. However, it is not generalized morphic because $r(2+2 g)$ is not cyclical generated. Note that over right generalized morphic rings $R$, each $R / R a$ have a right $\mathbb{R}$-resolution (see Lemma 3.2). In this section, over right generalized 
morphic rings, the $\mathrm{P}$-injective and $\mathrm{P}$-flat dimensions are investigated by some derived functors.

If $R$ is a right $(m, n)$-coherent ring, then $\operatorname{Hom}(-,-)$ is left balanced on ${ }_{R} \mathcal{M} \times{ }_{R} \mathcal{M}$ by $\mathscr{F}_{(m, n)} \times \mathscr{F}_{(m, n)}$. We let $\operatorname{Ex}_{t}(-,-)$ denote the left balance derived functors, and $\mathscr{F}_{(m, n)}^{\perp}=\left\{C \in_{R} \mathcal{M}: \operatorname{Ext}^{1}(F, C)=0, \forall F \in \mathscr{F}_{(m, n)}\right\}$ (in particular, a left $R$-module $C$ is called Warfield cotorsion (see [9] and [10]) provided that $\operatorname{Ext}^{1}(F, C)=0$ for every P-flat left $R$-module $F$ ).

Proposition 3.1. Let $R$ be a right $(m, n)$-coherent ring and $t \geq 2$. Then the following are equivalent for a left $R$-module $N$.

(1) left $\mathscr{F}_{(m, n)}-\operatorname{dim} N \leq t$.

(2) $\operatorname{Ex}_{t+k}(M, N)=0$ for all $M \in_{R} \mathcal{M}$ and all $k \geq-1$.

(3) $\operatorname{Ex}_{t-1}(M, N)=0$ for all $M \in_{R} \mathcal{M}$.

(4) $\operatorname{Ex}_{t-1}(M, N)=0$ for all left $R$-module $M \in \mathscr{F}_{(m, n)}^{\perp}$.

Proof. $(1) \Rightarrow(2) \Rightarrow(3) \Rightarrow(4)$ is trivial.

$(4) \Rightarrow(1)$. Let $\cdots \rightarrow F_{1} \rightarrow F_{0} \rightarrow N \rightarrow 0$ be a minimal left $\mathscr{F}_{(m, n)^{-}}$ resolution and $C=\operatorname{ker}\left(F_{t-1} \rightarrow F_{t-2}\right)$. Then $C \in \mathscr{F} \frac{\perp}{(m, n)}$ by [16, Lemma 2.1.1], and $\operatorname{Ex}_{t-1}(C, N)=0$ by (4). So we get that $C$ is isomorphic to a direct summand of $F_{t}$. Thus $C \in \mathscr{F}_{(m, n)}$, as desired.

Lemma 3.2. If $R$ is right generalized morphic and $a \in R$, then there is a right $\mathscr{F}$-resolution:

$$
0 \rightarrow R / R a \rightarrow R_{0} \rightarrow R_{1} \rightarrow \cdots \rightarrow R_{n} \rightarrow \cdots
$$

where $R_{i}=R, i=0,1,2, \ldots$ and each cokernel is cyclical presented (We call that right $\mathbb{R}$-resolution).

Proof. By [12, Theorem 3.1], there is an $\mathscr{F}$-preenvelope of $R / R a: f: R / R a \rightarrow$ $R^{t}$. Set $f(1+R a)=\left(s_{i}\right), s_{i} \in R$. Thus $s_{i} \in r(a)$. But $r(a)=b R$ for some $b \in R$. Then $s_{i}=b r_{i}$ for some $r_{i} \in R$. Define $g: R / R a \rightarrow R$ via $g(1+R a)=b$ and $h: R \rightarrow R^{t}$ via $h(1)=\left(r_{i}\right)$. Clearly, they are well-defined and $f=h g$. This means that $g$ is an $\mathscr{F}$-preenvelope of $R / R a$ and $\operatorname{coker}(g)=R / R b$. If repeat this procedure, we get the desired right $\mathbb{R}$-resolution of $R / R a$.

Proposition 3.3. Let $R$ be a right generalized morphic ring and $t \geq 2$. Then the following are equivalent for any $a \in R$.

(1) right $\mathscr{F}-\operatorname{dim} R / R a \leq t-2$.

(2) $\operatorname{Ex}_{t+k}(R / R a, N)=0$ for all $N \in_{R} \mathcal{M}$ and all $k \geq-1$.

(3) $\operatorname{Ex}_{t-1}(R / R a, N)=0$ for all $N \in \in_{R} \mathcal{M}$.

(4) $\operatorname{Ex}_{t-1}(R / R a, R / R b)=0$ for any $b \in R$.

(5) right $\mathcal{P}$ roj-dim $R / R a \leq t-2$.

(6) $H$ is a direct summand of $R_{t-2}$ for any right $\mathbb{R}$-resolution of $R / R a$, where $H=\operatorname{ker}\left(R_{t-2} \rightarrow R_{t-1}\right)$.

Proof. (1) $\Rightarrow(2) \Rightarrow(3) \Rightarrow(4)$ and (5) $\Rightarrow(1)$ are trivial. 
$(4) \Rightarrow(6)$. By Lemma 3.2, there is a right $\mathscr{F}$-resolution: $0 \rightarrow R / R a \rightarrow R_{0} \rightarrow$ $R_{1} \rightarrow \cdots$ with $R_{i}=R, i=0,1,2, \ldots$ and each cokernel is cyclical presented. Let $C=\operatorname{coker}\left(R_{t-2} \rightarrow R_{t-1}\right)$. Then $\operatorname{Ex}_{t-1}(R / R a, C)=0$ by (4). This implies that $C$ is isomorphic to a direct summand of $R_{t-1}$, and it is projective. Thus $D=\operatorname{Im}\left(R_{t-2} \rightarrow R_{t-1}\right)$ is projective, and so is $H=\operatorname{ker}\left(R_{t-2} \rightarrow R_{t-1}\right)$. It follows that $H$ is a direct summand of $R_{t-2}$,

$(6) \Rightarrow(5)$. Let

$$
0 \longrightarrow R / R a \stackrel{d_{0}}{\longrightarrow} R_{0} \stackrel{d_{1}}{\longrightarrow} R_{1} \longrightarrow \cdots \longrightarrow R_{t-1} \stackrel{d_{t}}{\longrightarrow} R_{t} \longrightarrow \cdots
$$

be a right $\mathbb{R}$-resolution of $R / R a, A=\operatorname{Im}\left(R_{t-3} \rightarrow R_{t-2}\right)$ and $H=\operatorname{ker}\left(R_{t-2} \rightarrow\right.$ $\left.R_{t-1}\right)$. Hence $R_{t-2}=H \oplus H^{\prime}$ for some left $R$-module $H^{\prime}$ by (6). Set $i$ : $H / A \rightarrow H / A \oplus H^{\prime}$ be an injection, $\pi: H / A \oplus H^{\prime} \rightarrow H / A$ be a projection and $\overline{d_{t-1}}: R_{t-2} / A \rightarrow R_{t-1}$ be the induced homomorphism of $d_{t-1}$. We claim that $\overline{d_{t-1}} i$ is an $\mathscr{F}$-preenvelope of $H / A$. In fact, for any left $R$-module $G \in \mathscr{F}$ and any homomorphism $g: H / A \rightarrow G$, there is $h: G \rightarrow R_{t-1}$ such that $g \pi=h \overline{d_{t-1}}$, whence $g=h\left(\overline{d_{t-1}} i\right)$. Note that $H=\operatorname{ker}\left(R_{t-2} \rightarrow R_{t-1}\right)$. Hence $\overline{d_{t-1}} i=0$, and so $0 \rightarrow R / R a \rightarrow R_{0} \rightarrow R_{1} \rightarrow \cdots \rightarrow R_{t-3} \rightarrow H \rightarrow 0$ is a right $\mathscr{F}$-resolution of $R / R a$.

Proposition 3.4. If $R$ is right $(m, n)$-coherent, then $-\otimes-$ on $\mathcal{M}_{R} \times_{R} \mathcal{M}$ is right balanced by $\mathscr{A}_{(m, n)} \times \mathscr{F}_{(m, n)}$.

Proof. If $G \in \mathscr{A}_{(m, n)}$, then $G^{+} \in \mathscr{F}_{(m, n)}$ by [17, Theorem 5.7]. For any left $R$-module $M$, by [12, Theorem 3.1] there is a right $\mathscr{F}_{(m, n)}$-resolution $0 \rightarrow M \rightarrow$ $F^{0} \rightarrow F^{1} \rightarrow \cdots$, and then $\cdots \rightarrow \operatorname{Hom}\left(F^{0}, G^{+}\right) \rightarrow \operatorname{Hom}\left(M, G^{+}\right) \rightarrow 0$ is exact. This means that $0 \rightarrow G \otimes M \rightarrow G \otimes F^{0} \rightarrow G \otimes F^{1} \rightarrow \cdots$ is exact.

Conversely, if $F \in \mathscr{F}_{(m, n)}$, then $F^{+} \in \mathscr{A}_{(m, n)}$. For any right $R$-module $N$, by $\left[12\right.$, Theorem 2.3], there is a right $\mathscr{A}_{(m, n)}$-resolution $0 \rightarrow N \rightarrow G^{0} \rightarrow G^{1} \rightarrow \cdots$ which is exact. Hence $\cdots \rightarrow \operatorname{Hom}\left(G^{0}, F^{+}\right) \rightarrow \operatorname{Hom}\left(N, F^{+}\right) \rightarrow 0$ is exact, which means that $0 \rightarrow N \otimes F \rightarrow G^{0} \otimes F \rightarrow G^{1} \otimes F \rightarrow \cdots$ is exact.

Now, we let $\mathrm{T}^{t}(-,-)$ denote the right balance derived functors of $-\otimes-$ on $\mathcal{M}_{R} \times_{R} \mathcal{M}$ by $\mathscr{A}_{(m, n)} \times \mathscr{F}_{(m, n)}$.

Lemma 3.5. $\mathscr{A}_{(m, n)}$ is closed under $(n, m)$-pure submodules.

Proof. Let $M \in \mathscr{A}_{(m, n)}$ and $N$ be an $(n, m)$-pure submodule of $M$. Then $M$ is $(n, m)$-pure in its injective envelope $E(M)$ by [19, Theorem 2.2]. And so $N$ is $(n, m)$-pure in $E(M)$ by [19, Proposition 2.9]. Consider the following sequence: $\operatorname{Hom}(P, E(M)) \rightarrow \operatorname{Hom}(P, N) \rightarrow \operatorname{Ext}^{1}(P, N) \rightarrow \operatorname{Ext}^{1}(P, E(M))$, where $P$ is $(m, n)$-presented, we get that $\operatorname{Ext}^{1}(P, N)=0$. Hence $N \in \mathscr{A}_{(m, n)}$.

Lemma 3.6 ([8, Lemma 8.4.23]). Let $N$ be a right $R$-module. If $M_{1} \rightarrow M_{2} \rightarrow$ $M_{3} \rightarrow M_{4}$ is an exact sequence of left $R$-modules such that $N \otimes M_{1} \rightarrow N \otimes M_{2} \rightarrow$ $N \otimes M_{3} \rightarrow N \otimes M_{4}$ is exact, then $0 \rightarrow N \otimes K \rightarrow N \otimes M_{3}$ is exact, where $K=\operatorname{ker}\left(M_{3} \rightarrow M_{4}\right)$. 
Proposition 3.7. Let $R$ be a right $(m, n)$-coherent ring and $t \geq 2$. Then the following are equivalent for a right $R$-module $M$.

(1) right $\mathscr{A}_{(m, n)}-\operatorname{dim} M \leq t$.

(2) $\mathrm{T}^{t+k}(M, N)=0$ for all $N \in_{R} \mathcal{M}$ and all $k \geq-1$.

(3) $\mathrm{T}^{t}(M, N)=\mathrm{T}^{t-1}(M, N)=0$ for any $N \in_{R} \mathcal{M}$.

(4) $\mathrm{T}^{t}(M, P)=\mathrm{T}^{t-1}(M, P)=0$ for any $(n, m)$-finitely presented $P \in_{R} \mathcal{M}$.

Proof. $(1) \Rightarrow(2)$. Let $0 \rightarrow M \rightarrow G^{0} \rightarrow G^{1} \rightarrow \cdots \rightarrow G^{t} \rightarrow 0$ be a right $\mathscr{A}_{(m, n)^{-}}$ resolution of $M$. Then $G^{t-2} \otimes N \rightarrow G^{t-1} \otimes N \rightarrow G^{t} \otimes N \rightarrow 0$ is exact and so $\mathrm{T}^{t}(M, N)=\mathrm{T}^{t-1}(M, N)=0$. But clearly $\mathrm{T}^{t+k}(M, N)=0$ for all $k \geq 1$. Hence (2) holds.

$(2) \Rightarrow(3) \Rightarrow(4)$ is trivial.

$(4) \Rightarrow(1)$. Let $0 \rightarrow M \rightarrow G^{0} \rightarrow G^{1} \rightarrow \cdots$ be a right $\mathscr{A}_{(m, n)}$-resolution of $M$. Then for any $(n, m)$-finitely presented left $R$-module $P, G^{t-2} \otimes P \rightarrow$ $G^{t-1} \otimes P \rightarrow G^{t} \otimes P \rightarrow G^{t+1} \otimes P$ is exact. Hence $K=\operatorname{ker}\left(G^{t} \rightarrow G^{t+1}\right)$ is $(n, m)$-pure in $G^{t}$ by Lemma 3.6, and so $K \in \mathscr{A}_{(m, n)}$ by Lemma 3.5. It follows that $0 \rightarrow M \rightarrow G^{0} \rightarrow G^{1} \rightarrow \cdots \rightarrow G^{t-1} \rightarrow K \rightarrow 0$ is a right $\mathscr{A}_{(m, n)}$-resolution of $M$.

We state [13, Propositions 4.13 and 4.14] or [18, Lemmas 3.6 and 3.8] as lemmas below.

Lemma 3.8. Let $R$ be a right generalized morphic ring and $t$ a fixed nonnegative integer. The following are equivalent for a right $R$-module $M$ :

(1) right $\mathscr{A}-\operatorname{dim} M \leq t$;

(2) $\operatorname{Ext}^{t+k}(R / a R, M)=0$ for every $a \in R$ and every $k \geq 1$;

(3) $\operatorname{Ext}^{t+1}(R / a R, M)=0$ for every $a \in R$;

(4) If $0 \rightarrow M \rightarrow G^{0} \rightarrow G^{1} \rightarrow \cdots \rightarrow G^{t-1} \rightarrow H \rightarrow 0$ is exact with each $G^{i}$ $P$-injective, then $H$ is P-injective.

Lemma 3.9. Let $R$ be a right generalized morphic ring and $t$ a fixed nonnegative integer. The following are equivalent for a left $R$-module $N$ :

(1) left $\mathscr{F}-\operatorname{dim} N \leq t$;

(2) $\operatorname{Tor}_{t+k}(R / a R, N)=0$ for every $a \in R$ and every $k \geq 1$;

(3) $\operatorname{Tor}_{t+1}(R / a R, N)=0$ for every $a \in R$;

(4) If $0 \rightarrow K \rightarrow F_{t-1} \rightarrow \cdots \rightarrow F_{1} \rightarrow F_{0} \rightarrow N \rightarrow 0$ is exact with each $F_{i}$ $P$-flat, then $K$ is P-flat.

Proposition 3.10. Let $R$ be a right generalized morphic ring and $t \geq 2$. Then the following are equivalent for any $a \in R$.

(1) right $\mathscr{F}-\operatorname{dim} R / R a \leq t-2$.

(2) $\mathrm{T}^{t+k}(M, R / R a)=0$ for all $M \in \mathcal{M}_{R}$ and all $k \geq-1$.

(3) $\mathrm{T}^{t}(M, R / R a)=\mathrm{T}^{t-1}(M, R / R a)=0$ for all $M \in \mathcal{M}_{R}$.

(4) $\mathrm{T}^{t}(R / b R, R / R a)=\mathrm{T}^{t-1}(R / b R, R / R a)=0$ for any $b \in R$.

Proof. (1) $\Rightarrow(2) \Rightarrow(3) \Rightarrow(4)$ is trivial. 
$(4) \Rightarrow$ (1). Let $0 \rightarrow R / R a \rightarrow R_{0} \rightarrow R_{1} \rightarrow \cdots \rightarrow R_{t} \rightarrow \cdots$ be a right $\mathbb{R}$-resolution of $R / R a$. Then for any $b \in R, R / b R \otimes R_{t-2} \rightarrow R / b R \otimes R_{t-1} \rightarrow$ $R / b R \otimes R_{t} \rightarrow R / b R \otimes R_{t+1}$ is exact. Hence $K=\operatorname{ker}\left(R_{t} \rightarrow R_{t+1}\right)$ is $(1,1)$ pure in $F_{t}$. Note the exact sequence $\operatorname{Tor}_{1}\left(R / b R, R_{t}\right) \rightarrow \operatorname{Tor}_{1}\left(R / b R, R_{t} / K\right) \rightarrow$ $R / b R \otimes K \rightarrow R / b R \otimes R_{t}$. This implies that $R_{t} / K$ is P-flat, and so $K$ is P-flat by Lemma 3.9. But $R_{t-2} \rightarrow R_{t-1} \rightarrow K$ is exact. Therefore $L=\operatorname{ker}\left(R_{t-2} \rightarrow R_{t-1}\right)$ and $R_{t-2} / L$ are P-flat again by Lemma 3.9. Note that $0 \rightarrow R / R a \rightarrow R_{0} \rightarrow$ $R_{1} \rightarrow \cdots \rightarrow R_{t} \rightarrow \cdots$ is a right $\mathbb{R}$-resolution of $R / R a$. Hence $R_{t-2} / L$ is isomorphic to a direct summand of $R_{t-1}$. This means that $R_{t-2} / L$ is projective and $L$ is a direct summand of $R_{t-2}$. Thus $0 \rightarrow R / R a \rightarrow R_{0} \rightarrow R_{1} \rightarrow \cdots \rightarrow$ $R_{t-3} \rightarrow L \rightarrow 0$ is a right $\mathscr{F}$-resolution of $R / R a$ by Proposition 3.3.

If $R$ is right $(m, n)$-coherent, then $\operatorname{Hom}(-,-)$ is left balanced on $\mathcal{M}_{R} \times \mathcal{M}_{R}$ by $\mathscr{A}_{(m, n)} \times \mathscr{A}_{(m, n)}$. We let $\mathrm{E}_{t}(-,-)$ denote the left balance derived functors.

Proposition 3.11. Let $R$ be a right $(m, n)$-coherent ring and $t \geq 2$. Then the following are equivalent for a right $R$-module $M$.

(1) right $\mathscr{A}_{(m, n)}-\operatorname{dim} M \leq t$.

(2) $\mathrm{E}_{t+k}(M, N)=0$ for all $N \in \mathcal{M}_{R}$ and all $k \geq-1$.

(3) $\mathrm{E}_{t-1}(M, N)=0$ for all $N \in \mathcal{M}_{R}$.

Proof. $(1) \Rightarrow(2) \Rightarrow(3)$ is trivial.

$(3) \Rightarrow(1)$. Let $0 \rightarrow M \rightarrow F^{0} \rightarrow F^{1} \cdots$ be a right $\mathscr{A}_{(m, n)}$-resolution of $M$ and $C=\operatorname{coker}\left(F^{t-2} \rightarrow F^{t-1}\right)$. Then $\mathrm{E}_{t-1}(M, C)=0$. So we get that $C$ is isomorphic to a direct summand of $F^{t}$ and $C \in \mathscr{A}_{(m, n)}$, as desired.

Recall that a right $R$-module $M$ is called reduced (see [8]) if $M$ has no nonzero injective submodules. $M$ is called D-injective ([13, Definition 3.1]) if $\operatorname{Ext}^{1}(G, M)=0$ for every P-injective right $R$-module $G$.

Proposition 3.12. Let $R$ be a right generalized morphic ring and $t \geq 2$. Then the following are equivalent for a right $R$-module $N$.

(1) left $\mathscr{A}-\operatorname{dim} N \leq t-2$.

(2) $\mathrm{E}_{t+k}(M, N)=0$ for all $M \in \mathcal{M}_{R}$ and all $k \geq-1$.

(3) $\mathrm{E}_{t-1}(M, N)=0$ for all reduced $D$-injective right $R$-module $M$.

Proof. $(1) \Rightarrow(2) \Rightarrow(3)$ is trivial.

$(3) \Rightarrow(1)$. If $A$ is D-injective, by Proposition $2.4, A$ has an $\mathscr{A}$-cover $f$ :

$F \rightarrow M$. There is an exact sequence $0 \longrightarrow F \stackrel{i}{\longrightarrow} E \longrightarrow L \longrightarrow 0$ with $E$ injective. Thus $L$ is P-injective by Lemma 3.8. Then there exists $g: E \rightarrow A$ such that $g i=f$, and so there exists $\varphi: E \rightarrow F$ such that $f \varphi=g$ since $f$ is a cover. Thus $f \varphi i=f$ and $\varphi i$ is an isomorphism. It follows that $F$ is isomorphic to a direct summand of $E$, and so $F$ is injective. From that, we get the minimal left $\mathscr{A}$-resolution of $N: \cdots \rightarrow F_{1} \rightarrow F_{0} \rightarrow N \rightarrow 0$, where each $F_{i}$ $(i \geq 1)$ is injective. Let $C=\operatorname{ker}\left(F_{t-1} \rightarrow F_{t-2}\right)$. Then $C$ is reduced D-injective by [13, Proposition 3.4], and $\mathrm{E}_{t-1}(C, N)=0$ by (3). Hence $C$ is isomorphic 
to a direct summand of $F_{t}$, that is, $C$ is injective, whence $C=0$. This means that $F_{t-1} \rightarrow F_{t-2}$ is injection. Note that $F_{t-2} \rightarrow H$ is an $\mathscr{A}$-cover of $H$, where $H=\operatorname{ker}\left(F_{t-3} \rightarrow F_{t-4}\right)$. This implies that $F_{t-1}=0$, as desired.

Remark 3.13. From the proof of Proposition 3.12, we get that if $R$ is right strongly P-coherent, then Proposition 3.12 holds by [13, Proposition 4.13 and Proposition 3.4]. Hence, conditions (1) and (2) of [13, Theorem 4.12] are equivalent.

Theorem 3.14. Let $R$ be a right generalized morphic ring and $t \geq 2$. Then the following are equivalent.

(1) gl right $\mathscr{A}-\operatorname{dim} \mathcal{M}_{R} \leq t$.

(2) gl left $\mathscr{F}-\operatorname{dim}_{R} \mathcal{M} \leq t$.

(3) gl left $\mathscr{A}-\operatorname{dim} \mathcal{M}_{R} \leq t-2$.

(4) right $\mathscr{F}-\operatorname{dim} R / R b \leq t-2$ for all $b \in R$.

(5) right $\mathscr{A}-\operatorname{dim} R / a R \leq t$ for all $a \in R$.

(6) right $\mathscr{A}-\operatorname{dim} M \leq t$ for all reduced $D$-injective right $R$-module $M$.

(7) $\operatorname{Ex}_{t-1}(M, N)=0$ for all $M, N \in \in_{R} \mathcal{M}$ and all $k \geq-1$.

(8) $\operatorname{Ex}_{t-1}(M, N)=0$ for all $M, N \in_{R} \mathcal{M}$.

(9) $\operatorname{Ex}_{t-1}(M, N)=0$ for all $N \in \in_{R} \mathcal{M}$ and all Warfield cotorsion left $R$ module $M$.

(10) $\operatorname{Ex}_{t-1}(R / R a, N)=0$ for all $N \in \in_{R} \mathcal{M}$ and all $a \in R$.

(11) $\operatorname{Ex}_{t-1}(R / R a, R / R b)=0$ for all $a, b \in R$.

(12) $\mathrm{T}^{t+k}(M, N)=0$ for all $M \in \mathcal{M}_{R}, N \in_{R} \mathcal{M}$ and all $k \geq-1$.

(13) $\mathrm{T}^{t}(M, N)=\mathrm{T}^{t-1}(M, N)=0$ for all $M \in \mathcal{M}_{R}, N \in \in_{R} \overline{\mathcal{M}}$.

(14) $\mathrm{T}^{t}(R / a R, N)=\mathrm{T}^{t-1}(R / a R, N)=0$ for all $N \in_{R} \mathcal{M}$ and all $a \in R$.

(15) $\mathrm{T}^{t}(M, R / R b)=\mathrm{T}^{t-1}(M, R / R b)=0$ for all $M \in \mathcal{M}_{R}$ and all $b \in R$.

(16) $\mathrm{T}^{t}(R / a R, R / R b)=\mathrm{T}^{t-1}(R / a R, R / R b)=0$ for all $a, b \in R$.

(17) $\mathrm{E}_{t+k}(M, N)=0$ for all $M, N \in \mathcal{M}_{R}$ and all $k \geq-1$.

(18) $\mathrm{E}_{t-1}(M, N)=0$ for all reduced $D$-injective right $R$-module $M$ and all $N \in \mathcal{M}_{R}$.

(19) $\mathrm{E}_{t-1}(R / a R, M)=0$ for all right $R$-module $M$ and all $a \in R$.

(20) right Proj-dim $R / a R \leq t-2$ for all $a \in R$.

(21) $H$ is a direct summand of $R_{t-2}$ for any right $\mathbb{R}$-resolution of $R / R a$ and any $a \in R$, where $H=\operatorname{ker}\left(R_{t-2} \rightarrow R_{t-1}\right)$.

(22) $\operatorname{Ext}^{t+1}(R / a R, M)=0$ for all $M \in \mathcal{M}_{R}$ and all $a \in R$.

(23) $\operatorname{Tor}_{t+1}(R / a R, N)=0$ for all $N \in \in_{R} \mathcal{M}$ and all $a \in R$.

(24) $\mathrm{fd}(R / a R) \leq t$.

(25) $\operatorname{pd}(R / a R) \leq t$.

Proof. (1) $\Leftrightarrow(2) \Leftrightarrow(22) \Leftrightarrow(23) \Leftrightarrow(24) \Leftrightarrow$ (25) comes from [13, Theorem $4.15]$ or $[18$, Theorem 3.11]. (2) $\Leftrightarrow(7) \Leftrightarrow(8) \Leftrightarrow(9)$ and $(4) \Leftrightarrow(10) \Leftrightarrow(11) \Leftrightarrow$ $(20) \Leftrightarrow(21)$ hold by Propositions 3.1 and 3.3 , respectively. (1) $\Leftrightarrow(12) \Leftrightarrow$ $(13) \Leftrightarrow(15),(16) \Leftrightarrow(5) \Leftrightarrow(14)$ and $(15) \Leftrightarrow(4) \Leftrightarrow(16)$ hold by Propositions 
3.7 and 3.10. (1) $\Leftrightarrow(3) \Leftrightarrow(17) \Leftrightarrow(18) \Leftrightarrow(6)$ and (5) $\Leftrightarrow$ (19) follow by Propositions 3.11 and 3.12 .

When $t=0$, we have:

Proposition 3.15. The following are equivalent for any ring $R$.

(1) $R$ is von Neumann regular.

(2) $R$ is a right generalized morphic and left $P$-injective ring with right $\mathscr{F}$ $\operatorname{dim} R / R b<\infty$ for any $b \in R$.

(3) gl right $\mathscr{A}-\operatorname{dim} \mathcal{M}_{R}=0$.

Proof. (1) $\Leftrightarrow(3)$ and $(1) \Rightarrow(2)$ are clear.

$(2) \Rightarrow(1)$. Since $R$ is P-injective, $R^{+}$is P-flat by Corollary 2.6. In terms of (2) and Corollary 2.2, there exists an exact right $\mathscr{F}$-resolution: $0 \rightarrow R / R a \rightarrow$ $F^{0} \rightarrow F^{1} \rightarrow \cdots \rightarrow F^{t} \rightarrow 0$ for any $a \in R$. This implies that $R / R a$ is $\mathrm{P}$-flat by Lemma 3.9, and so $R / R a$ is isomorphic to a direct summand of $R$. Thus $R / R a$ is projective and $R a$ is a direct summand of $R$. Hence, $R$ is von Neumann regular.

Recall that a ring $R$ is called right p.p. in case principal right ideals are all projective. When $t=1$, we have:

Proposition 3.16. The following are equivalent for any ring $R$.

(1) $R$ is a right p.p. ring.

(2) $R$ is a right generalized morphic ring with gl right $\mathscr{A}-\operatorname{dim} \mathcal{M}_{R} \leq 1$.

(3) $R$ is right generalized morphic and every left $R$-module has an epic $P$-flat envelope.

(4) $R$ is right generalized morphic and $R / R a$ has an epic $P$-flat envelope for any $a \in R$.

(5) For any $a \in R, r(a)=b R$ and $R b$ is projective for some $b \in R$.

In this case, for any $a \in R$, if $r(a)=c R$ for some $c \in R$, then $R c$ is projective and $R / R a \rightarrow c R, 1+R a \mapsto c$ is an epic P-flat envelope of $R / R a$.

Proof. (1) $\Leftrightarrow(2)$ follows by [13, Theorem 5.3]. (1) $\Leftrightarrow$ (3) holds since (1) equivalent to that every submodule P-flat left $R$-module is P-flat by [18, Corollary 3.12 . (3) $\Rightarrow(4)$ is clear.

$(4) \Rightarrow(1)$. Let $N$ be a submodule of a P-flat left $R$-module $M$. For any $a \in R$ and any homomorphism $f: R / R a \rightarrow N$, there is a free left $R$-module $F, g: R / R a \rightarrow F$ and $h: F \rightarrow M$ such that $i f=h g$, where $i: N \rightarrow M$ is an inclusion map. By (4), $R / R a$ has an epic P-flat envelope $\alpha: R / R a \rightarrow P$. Then there is $\beta: P \rightarrow F$ such that $g=\beta \alpha$. Thus if $=(h \beta) \alpha$, whence $\operatorname{ker}(\alpha) \subseteq \operatorname{ker}(f)$. Define $s: P \rightarrow N$ via $s(\alpha(x))=f(x)$ for any $x \in R / R a$. It is clear that $s$ is well-defined and $f=s \alpha$. This shows that $N$ is P-flat and (1) holds.

$(4) \Rightarrow(5)$. For any $a \in R$, by (4), $R / R a$ has an epic P-flat envelope $f$ : $R / R a \rightarrow F$. Note that there exists a P-flat preenvelope of $R / R a g: R / R a \rightarrow R$ via $g(1+R a)=b, r(a)=b R$ by Lemma 3.2. Then there exist $h: R \rightarrow F$ and 
$\varphi: F \rightarrow R$ such that $f=h g$ and $g=\varphi f$. Thus $f=h \varphi f$, and so $h \varphi=i d$ since $f$ is epic. It follows that $F$ is projective. Since $g=\varphi f, \operatorname{Im}(g)=\operatorname{Im}(\varphi)=R b$, whence $F \cong R b$ and $R b$ is projective.

$(5) \Rightarrow(4)$. Clearly, $R$ is right generalized morphic. By Lemma 3.2, we can define a P-flat preenvelope of $R / R a f: R / R a \rightarrow R$ such that $\operatorname{Im}(f)=R b$. Since $R b$ is projective by (5), $R / R a \rightarrow R b$ is an epic P-flat envelope.

If $r(a)=c R$ for some $c \in R$, by Lemma 3.2, there exists a P-flat preenvelope of $R / R a g: R / R a \rightarrow R$ via $g(1+R a)=c$. From the proof of $(4) \Rightarrow(5)$, we get that $R c$ is projective and $R / R a \rightarrow c R, 1+R a \mapsto c$ is an epic P-flat envelope of $R / R a$.

When $t=2$, we have:

Proposition 3.17. The following are equivalent for any ring $R$.

(1) For any $a \in R, r(a)$ is cyclical generated and projective.

(2) For any $a \in R, r(a)$ is isomorphic to a direct summand of $R$.

(3) $R$ is right generalized morphic and $R / R a$ has a P-flat envelope with unique mapping property for any $a \in R$.

(4) $R$ is a right generalized morphic ring with gl right $\mathscr{A}-\operatorname{dim} \mathcal{M}_{R} \leq 2$.

(5) For any $a \in R$, there exists $b \in R$ such that $r(a)=b R$, and projective left $R$-modules $P, Q$ such that $b \in P, R=P \oplus Q$ and $r(b) \cap r(Q)=0$.

In this case, if $r(a)=b R$ or $r(a)=c R$ for some $b, c \in R$, then $R c \cong R b$.

Proof. (1) $\Leftrightarrow(2)$ is trivial and (1) $\Leftrightarrow(3) \Leftrightarrow$ (4) follows form Theorem 3.14.

$(3) \Rightarrow(5)$. Let $f: R / R a \rightarrow P$ be a P-flat envelope with unique mapping property. By Lemma 3.2, there exists a P-flat preenvelope of $R / R a g: R / R a \rightarrow$ $R$ with $g(1+R a)=b, r(a)=b R$ for some $b \in R$. Then there are $h: P \rightarrow R$ and $\alpha: R \rightarrow P$ such that $g=h f$ and $f=\alpha g$. Thus $f=\alpha h f$, and hence $R=h(P) \oplus Q$ for some projective left $R$-module $Q$. We identify $P$ with $h(P)$. Thus $b \in P$.

Now we let $P=R p$ and $Q=R(1-p)$ for some idempotent $p \in R$. Let $s \in r(b) \cap r(Q)$. Define $\beta: P \rightarrow R$ via $\beta(p)=s$. Clearly, $\beta$ is well-defined. But we see that $\beta f(1+R a)=\beta(b)=\beta(b p)=b s=0$, that is, $\beta f=0$. Since $f$ is an envelope with unique mapping property, $\beta=0$. It follows that $s=0$.

$(5) \Rightarrow(3)$. Clearly, $R$ is right generalized morphic. By (5), it is easy to check that $f: R / R a \rightarrow P$ via $f(1+R a)=b$ is a P-flat preenvelope of $R / R a$. Let $P=R p$ and $Q=R(1-p)$ for an idempotent $p \in P$. If $g: P / R b \rightarrow R^{n}$ is a P-flat preenvelope of $P / R b$. Set $g(p+R b)=\left(s_{i}\right), s_{i} \in R$. Since $b p=b$ and $q p=0$ for any $q \in Q$, each $s_{i} \in r(b) \cap r(Q)$. By (4), we have $s_{i}=0$. Hence $0 \rightarrow \operatorname{Hom}(P, G) \rightarrow \operatorname{Hom}(R / R a, G) \rightarrow 0$ is exact for any P-flat left $R$-module $G$, and so (3) holds.

From the proof of $(3) \Rightarrow(5)$, we get that if $r(a)=b R$ or $r(a)=c R$ for some $b, c \in R$, then there are two direct summands of $R: P$ and $P_{1}$ such that $R / R a \rightarrow P(1+R a \mapsto b)$ and $R / R a \rightarrow P_{1}(1+R a \mapsto c)$ are envelopes. It follows that $R c \cong R b$. 


\section{References}

[1] F. W. Anderson and K. R. Fuller, Rings and Categries of Modules, Second edition, Springer-Verlag, Berlin, 1974.

[2] T. J. Cheatham and D. R. Stone, Flat and projective character modules, Proc. Amer. Math. Soc. 81 (1981), no. 2, 175-177.

[3] J. L. Chen and N. Q. Ding, The weak global dimension of commutative coherent rings, Comm. Algebra 21 (1993), no. 10, 3521-3528.

[4] J. L. Chen, N. Q. Ding, Y. L. Li, and Y. Q. Zhou, On $(m, n)$-injectivity of modules, Comm. Algebra 29 (2001), no. 12, 5589-5603.

[5] R. R. Colby, Rings which have flat injective modules, J. Algebra 35 (1975), 239-252.

[6] N. Q. Ding, On envelopes with the unique mapping property, Comm. Algebra 24 (1996), no. 4, 1459-1470

[7] E. E. Enochs, Injective and flat covers, envelopes and resolvents, Israel J. Math. 39 (1981), no. 3, 189-209.

[8] E. E. Enochs and O. M. G. Jenda, Relative Homological Algebra, de Gruyter Exp. Math., vol 30, de Gruyter Berlin, 2000.

[9] L. Fuchs and L. Salce, Modules over Non-Noetherian Domains, Math. Surveys and Monographs. Vol. 84. Providence, Amer. Math. Society, 2001.

[10] R. Göbel and J. Trlifaj, Approximations and Endomorphism Algebras of Modules, GEM 41. Berlin-New York, Walter de Gruyter, 2006.

[11] H. Holm and P. Jørgensen, Covers, precovers and purity, Illinois J. Math. 52 (2008), no. 2, 691-703

[12] L. X. Mao and N. Q. Ding, On relative injective modules and relative coherent rings, Comm. Algebra 34 (2006), no. 7, 2531-2545.

[13] _ On divisible and torsionfree modules, Comm. Algebra 36 (2008), no. 2, 708-731.

[14] W. K. Nicholson and E. Sánchez Campos, Rings with the dual of the isomorphism theorem, J. Algebra 271 (2004), no. 1, 391-406.

[15] A. Shamsuddin, n-injective and n-flat modules, Comm. Algebra 29 (2001), no. 5, 20392050.

[16] J. Xu, Flat Covers of Modules, Lecture Notes in Math. 1634. Berlin-Heidelberg-New York, Springer-Verlag, 1996.

[17] X. X. Zhang and J. L. Chen, On $(m, n)$-injective modules and $(m, n)$-coherent rings, Algebra Colloq. 12 (2005), no. 1, 149-160.

[18] H. Y. Zhu and N. Q. Ding, Generalized morphic rings and their applications, Comm. Algebra 35 (2007), no. 9, 2820-2837.

[19] Z. Zhu, J. L. Chen, and X. X. Zhang, On $(m, n)$-purity of modules, East-West J. Math. 5 (2003), no. 1, 35-44.

YUEDI ZENG

Department of Mathematics

Putian College

Fujian, 351100, P. R. China

E-mail address: yuedizeng@gmail.com

JiANLONG CHEN

Department of Mathematics

Southeast University

NANJing, 210096, P. R. ChinA

E-mail address: jlchen@seu.edu.cn 